\title{
Digitization of art pieces based on 3D, colour and texture parameters
}

\author{
Jean-Pierre CHAMBARD, Vincent CHALVIDAN, Mohammed TAZEROUALTI ${ }^{\text {a }}$ \\ Frédéric LARUE, Jean-Michel DISCHLER ${ }^{\mathrm{b}}$ \\ Virginie VURPILLOT, Anne-Claire LEGRAND ${ }^{\mathrm{c}}$ \\ ${ }^{\mathrm{a}} \mathrm{HOLO} 3$ \\ ${ }^{\mathrm{b}}$ Le Laboratoire des Sciences de l'Image, de l'Informatique et de la Télédétection \\ ${ }^{c}$ Laboratoire d'Informatique Graphique et d'Ingénierie de la Vision
}

\begin{abstract}
Within the framework of a French research program, we have developed an optical system dedicated to the measurement of works of art. The purpose is to record the actual optical characteristics of the objects' surfaces in order to be able to display the art object on a screen with a high degree of realism. Three types of data are recorded: 3D shape, true colours and texture.

The 3D shape is obtained using a structured light sensor that gives a dense point cloud. A specific procedure allows automatic registration of several point clouds without any contact with the surface.

The colours maps are recorded with the structured light sensor's RGB camera and using a built-in lighting system. Both camera and lighting are calibrated using a well-defined procedure. Merging the colour data with the 3D data is straightforward because the same camera is used for both tasks.

The texture information is related to the so-called BRDF (Bi-directionnal reflectance distribution function): at each point on the surface, the reflectance is a function of the direction of observation and the direction of illumination. To record this complex texture information, several illumination sources are used, as well as an additional moving camera. Thus, for one 3D point cloud, a complete set of colour pictures is processed to produce texture files that are directly linked to the $3 \mathrm{D}$ points. presented.

The paper details the measurement procedure as well as the associated data processing. Several results are
\end{abstract}

Keywords : works of art, 3D digitization, reflectance, texture, BRDF, colour, calibration, registration

\section{INTRODUCTION}

Today heritage conservation is becoming increasingly important in all the developed nations. In the case of France alone, it is estimated that there are 3,625,000 works of art. On the other hand, the development of communication tools is leading to strong growth in the demand for images. Today, dissemination via the internet or CD-ROM is based on 2D images which are subject to copyright. The current price for the granting of rights to a $2 \mathrm{D}$ silver-emulsion based photograph is in the region of $300 €$. The transition from 2D to 3D photos represents an opportunity for the holders of works of art, and museums in particular, to set up an image bank that is exclusive and free from copyright. Images could then be sold, with a return on the investment.

3D scanning tools appeared on the market some years ago, based on the general principle of triangulation. This principle lets you obtain either a single point $\mathrm{X}, \mathrm{Y}, \mathrm{Z}$ (point triangulation), or a section giving a set of points $\mathrm{X}, \mathrm{Y}, \mathrm{Z}$ on a line 
(laser sheet triangulation), or a complete 3D surface (structured light principle). The general characteristic of these tools is their rapid acquisition (up to 40,000 points per second), the absence of contact with the object being measured and the automatic nature of their operation. Industrial applications for these sensors are firstly, surface scanning for CAD file reconstruction of old objects (reverse engineering), then more recently, dimensional measurement. Today therefore, these sensors are becoming true measurement tools.

Concerning applications in the field of works of art, we can cite the work of a Canadian laboratory belonging to the CNRC (Canadian National Research Council) in Ottawa which is combining measurement of relief by laser plane scanning, with colour measurement by laser scanning using 3 wavelengths [1]. This process is extremely effective in terms of acquisition speed because relief measurement is carried out simultaneously with colour measurement. However, colour and texture acquisition is only carried out in one specific lighting direction, which is that of the laser projection. It also has the drawbacks inherent in the use of lasers, such as safety and cost. A patented applied for by SIEMENS [2] also presents a system that enables both $3 \mathrm{D}$ and colour acquisition to be carried out at the same time, 3D acquisition being carried out using infrared lighting. In this case too, colour acquisition is only carried out for one lighting direction and the aspect of texture is not tackled.

The European CRISATEL project is proposing to implement a complex, multi-spectral acquisition system. This system, which was developed for flat works of art (paintings) requires careful set-up, very powerful lighting sources, as well as a calibration method specific to paintings (pigment calibration).

Information concerning texture is often acquired at the same time as that of geometry and colour [3]. It has long been neglected in favour of geometric and colorimetric precision. But its importance is crucial in the context of quality threedimensional restitution on a graphic display. Often, as is the case here, the capture system works in consecutive stages, supplying collections of images which need to be joined (an object is scanned in several parts, by rotation or translation). These slides are generally taken under variable conditions of view and lighting, creating discontinuity of texture when they are rebuilt using a process called texture stitching [4]. The fact that it is often difficult to isolate surface reflection prevents current systems from being able to display objects subsequently, under different lighting conditions. Unwanted characteristics, such as specular reflections, may appear and affect visual quality considerably during restitution if they are not handled correctly. Finally, the amount of data is far too great to allow display in real time on an average computer such as a PC, even if it has a high-performance graphics card. Reconstruction of detailed texture is therefore, a means of alleviating this difficulty [5].

In this paper, we present a process that enables all the optical and geometrical characteristics of a surface (colour data, texture and 3D shape) to be recorded in one and the same file, so that works of art can be restored in an extremely realistic manner on a computer screen.

\section{THE SCANNING EQUIPMENT}

The equipment is based on a 3D scanning sensor developed by HOLO3 for industrial applications and uses the structured light technique (fringe projection). Several changes and additions were made to this sensor for the purpose of recording colour and texture:

- the use of a colour camera in the 3D scanning sensor

- $\quad$ the addition of an additional mobile camera for capturing texture data

- the addition of diffuse lighting for recording colour

- $\quad$ the addition of point source lighting for the texture aspect.

After carrying out a study, we selected the PCO 2000 camera with a resolution of 2000x2000 pixels and a 14-bit dynamic range, combined with a CRI programmable RGB colour filter. Two types of sources were chosen to light the objects: CHIMERA diffuse lighting and a set of point source lighting based on SOLUX lamps.

The whole of this equipment was mounted on a trolley (see fig 1 below) and enabled us to make recordings on-site. The field scanned in one view is $600 \times 400 \mathrm{~mm}$. Spatial resolution is approximately $0.3 \mathrm{~mm}$. 


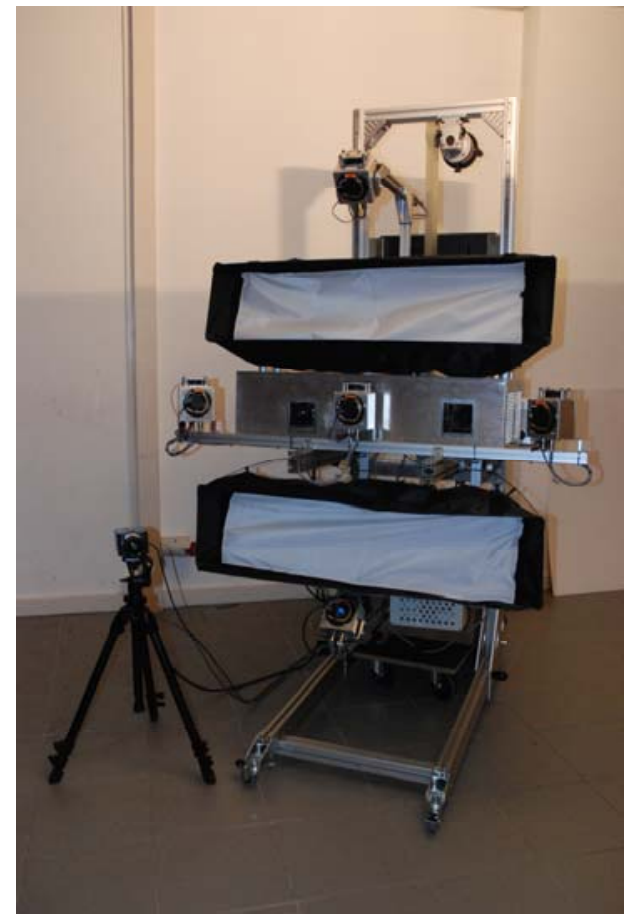

Fig. 1: Scanning equipment

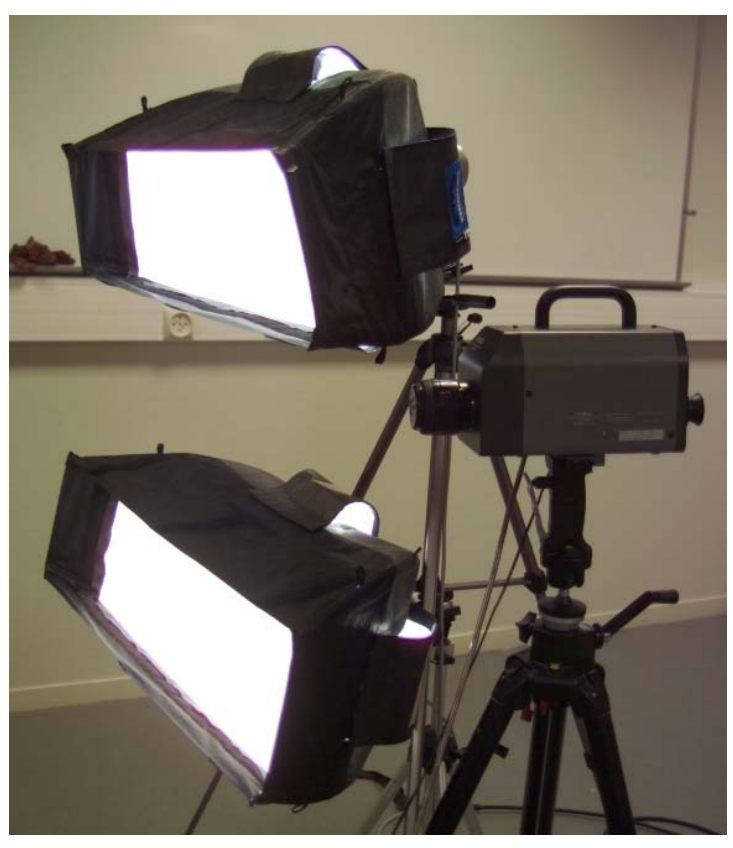

Fig. 2: Calibration of diffuse illumination 


\section{COLOUR ACQUISITION}

Characterization of the scanning system consists of determining the characteristics of each component: lighting (see fig 2), colour filter, optics and the sensor so as to define the properties and limits of the system, the ideal configurations and to determine how it needs to be calibrated. This work has enabled us to specify performance in terms of spatial resolution, acquisition speed, and precision according to the various possible configurations. At the end of this characterization phase, the scanning procedure was supplemented by adjustments to the configuration parameters such as optimization of integration time.

Calibrating the colour scanning system

Calibration of the scanning system is carried out in order to ensure that the works of art are scanned correctly and reliably. This phase consists of studying methods of colour calibration, overlapping the sensor's spectral sensitivity curves, calculating reference colour values, studying changes in colour spaces, choosing and validating the calibration algorithm, integrating the calibration procedure into the colour model and setting up functional validation tests. At the end of this phase, a colour calibration protocol has been inserted into the scanning protocol. This protocol enables raw colour data to be transformed into calibrated data and thus ensures faithful reproduction of the colours of the work that has been scanned. Measurement of the quality of the data measured was also set up, especially in terms of noise and over or under exposure, in order to ensure that the data acquired are usable within the context of the calibration procedure. Detail of this work is reported in [6-9]

\section{RECORDING THE 3D SHAPE}

The 3D scanning by fringe projection processes enable a large quantity of data (dense point cloud) to be obtained in less than a minute. However, it is generally necessary to display these point clouds in order to remove aberrant points that exist on the edge of objects or close to object discontinuities. This consists of long and painstaking manual work. We carried out an in-depth study for the purpose of improving the quality of 3D point clouds and removing all the scanning artefacts (phantom clouds). The purpose of the study was, on the one hand, to identify the origin of these artefacts and on the other hand, to correct them as far as possible.

\subsection{Cancelling digitization artefacts}

The main results of this study are summarized in the table below. Five different origins of scanning problems were identified. In each case, we were able to develop a suitable algorithm which permitted detection of these sources of noise and which removed the phantom points. Using specific adaptive filtering permitted in the end a reduction in spatial noise of the point clouds by a factor of 5 to 10 . The details of the algorithms can be found in [10].

The point clouds obtained in this way after automatic filtering are free from artefacts and can be used directly in the next phase which is the surface reconstruction. 


\begin{tabular}{|l|c|l|l|}
\hline Origin of the artefact & $\begin{array}{l}\text { Effect on the phase } \\
\text { (standard deviation } \sigma)\end{array}$ & Solution proposed & $\begin{array}{l}\text { Residual errors in phase } \\
\text { after correction }\end{array}$ \\
\hline Zone not lit & Phase jump of $2 \pi$ & $\begin{array}{l}\text { Threshold on modulations } \\
\text { ratio }\end{array}$ & $\sigma=5 \mathrm{mrad}$ \\
\hline $\begin{array}{l}\text { Phase jump on the object } \\
\text { surface }\end{array}$ & $\sigma=50 \mathrm{mrad}$ & idem & $\sigma=5 \mathrm{mrad}$ \\
\hline $\begin{array}{l}\text { Reflection from one surface } \\
\text { of the object to another }\end{array}$ & $\sigma=100 \mathrm{mrad}$ & idem & $\sigma=5 \mathrm{mrad}$ \\
\hline $\begin{array}{l}\text { Reflectance variation of the } \\
\text { surface }\end{array}$ & $\sigma=50 \mathrm{mrad}$ & $\begin{array}{l}\text { Threshold concerning } \\
\text { intensity variations }\end{array}$ & $\sigma=7 \mathrm{mrad}$ \\
\hline Entire projected sine value & $\sigma=100 \mathrm{mrad}$ & $\begin{array}{l}\text { To increase the Airy } \\
\text { projector task }\end{array}$ & $\sigma=1.7 \mathrm{mrad}$ \\
\hline
\end{tabular}

\subsection{Automatic registration of clouds}

We suggested a new protocol for the acquisition of 3D surfaces, a protocol which permits automatic mapping of several scanned views. A structured light pattern was used to project a parameterization onto surface fragments. Each surface point in intersection with this parameterization is identified individually, independently of the view point. Thus the problem of point-to-point and point-to-pixel mapping is immediately solved. This mapping is then used to carry out automatic joining of surfaces. This automatic registration process is described in detail in [11].

\section{THE RECORD AND DISPLAY OF TEXTURE}

We suggested a protocol for the directional and bi-directional acquisition of texture, this protocol implementing the mapping of several scanned views referred to above. In the case of directional acquisition, we associated a group of pixels from images obtained using an external mobile camera with each point of the 3D surface scanned. This enables us to identify the colour of the surface with precision, according to the observer's view point. The term generally used is "lightfield texture". We used a representation of this directional information based on a polynomial (according to the "polynomial textures" principle) in such a way as to permit real time restitution on the graphic screen. This representation has the advantage of being compact, but the drawback that it degrades information in cases of complex reflectance. We subsequently extended this principle to bi-directional textures by taking for each view point of the external mobile camera, a collection of images using variable lighting based on 5 separate lamps positioned precisely around the sensor. These supplementary images are obtained by successively blocking off 4 of the 5 lamps, enabling the lighting of one lamp at a time to be retained. The set of images lets you obtain groups of pixels for each surface point, the colour of which depends both on the position of the light source and that of the observer (hence the term bi-directional). This information is then represented in the form of "Lafortune lobes" in order to permit real time restitution on a graphic screen, here again, with possible deterioration of the quality of the information depending on the reflectance complexity. We also implemented a representation in spherical harmonics form where we showed that it could also apply to the real time display on a screen of a transparent and refracting scanned object. Finally, we suggested a new process permitting analysis of a texture for later virtual reproduction of the appearance of an object, something that would for example permit giving a CAD object an appearance similar to that of a scanned object. This work on texture is detailed in [12-15]. 


\section{EXAMPLES OF RESULTS}

The set of developments carried out came to fruition in a prototype sensor, combined with acquisition and restitution software. The 3D scanning and automatic registration processes enable a set of 3D points to be obtained describing an approximation of the surface of a real object. In addition to its position in space, colour information (which we call texture) is associated with each of these points, varying according to the observer's position and possibly according to the direction of the lighting. The scanned copy includes therefore, not only the geometric aspects of the object scanned, but also its visual appearance according to given conditions of observation and lighting. It is a question therefore of a faithful digital copy of the work, far more complex than a simple digital photograph. The object can therefore be interactively displayed on screen when changing observation and lighting parameters.

Fig 3 and 4 show two examples of reconstructed textured objects. The relatively small Greek vase is modelled using 5 different views for the $3 \mathrm{D}$ recording. The texture information corresponds to 27 viewpoints. The dataset (shape, colour and texture) is a file of $20 \mathrm{MB}$. The African wood statue is also properly reconstructed and it is possible to recognize the specific texture of wood. Both objects are relatively small compared to the field of view of the sensor.
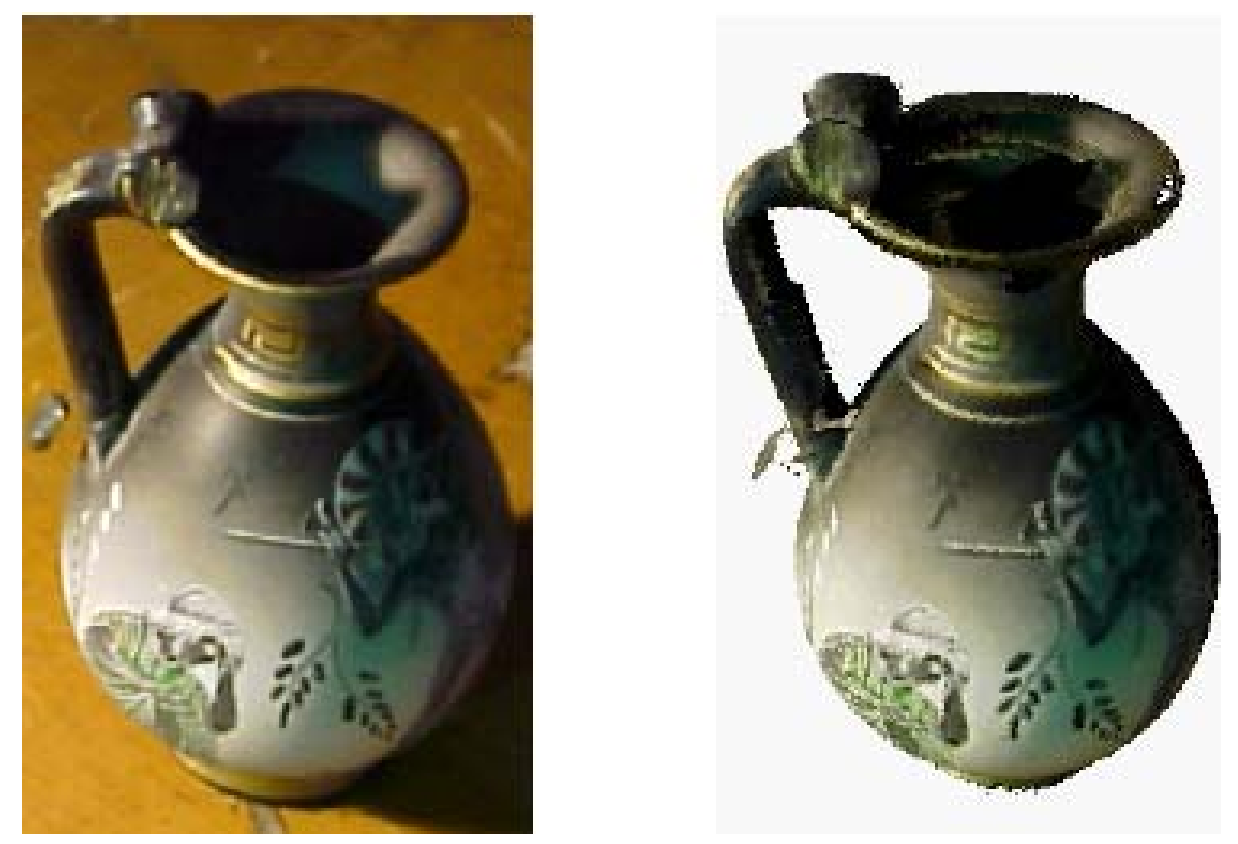

Fig. 3 : Left : a picture of the Greek vase model. Right : A synthetic view generated using "light field texture". All data are registered in a fully automatic manner. 

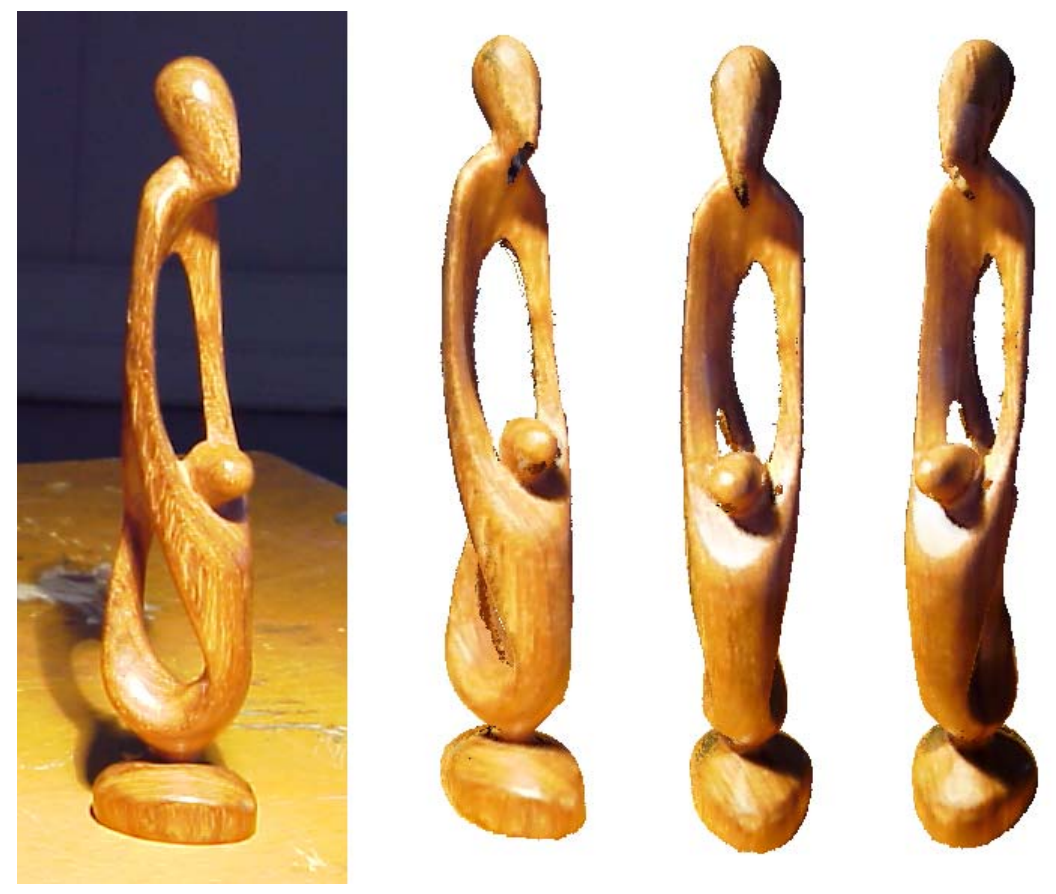

Fig.4 : Left : a picture of the wood statue. Right : Synthetic views of the object reconstructed with its texture

\section{CONCLUSION AND FUTURE PERSPECTIVES}

In this era of image communication, the production of high quality images of works of art is by nature set to increase the dissemination of cultural heritage quite significantly. In order to guarantee the quality restitution of a work of art on the screen, it is necessary to be able to reproduce all of this object's visual information. Of course this implies the colour, but also the relief and light environment of the work. We have obtained very encouraging results in this field, by integrating into the same item of equipment, and using a unique procedure, the recording of an object's colour, 3D shape and texture. The restitution of all this data on a computer screen permits a high degree of realism to be obtained in the display. One interesting aspect of this work is that the texture recorded and displayed is the actual texture of the object, including all artefacts or "defects" that this object may have. At the end, we get a faithful digital copy of the work of art. The whole of the data recording procedure is automatic. Manual operations are guided by software which means that the process can be used by a non-specialist.

Taking into account the rich amount of information recorded, the files generated are extremely large. In circumstances where a number of views are required in order to cover the whole object, the display loses its fluidity, and the movements become slow or jerky. Further optimization needs to be done in order to handle this type of object. 


\section{REFERENCES}

[1] International Patent WO 97/33139

[2] International Patent WO 00/14478

[3] P. Longère et A. Trémeau, "Color appearance : effects of texture and relief", International Symposium on Electronic Image Capture and Publishing, Vol. 3409, pp 89-97, Zurich, 1998.

[4] Rocchini C., Cignoni P., Montani C., "Multiple Textures Stitching and Blending on 3D Objects", Rendering Techniques, SpringerComputerScience, June 1999.

[5] F. Bernardini, I. Martin, H. Rushmeier, "High-Quality Texture Reconstruction from Multiple Scans", IEEE TVCG 7(4), october-december, 2001.

[6] V.Vurpillot, A.Legrand, A.Tremeau, "Color Calibration of an acquisition device", 2nd International Conference on Computer Vision Theory and Applications, VISAPP'07, Barcelone, Espagne, 8-11 mars 2007.

[7] V.Vurpillot, A.Legrand, A.Tremeau, "Spectral Sensitivity Estimation for Color Camera Calibration", CGIV'06, Third European Conference on Colour in Graphics, Imaging, and Vision, Leeds, UK, 19-22 Juin 2006.

[8] V.Vurpillot, A.Legrand, A.Tremeau, "Numérisation et authentification d'œuvres d'art, 3D, texture et couleur", Colloque Aratem'2006, Saint Etienne, France, 9 fevrier 2006

[9] V.Vurpillot, A.Legrand, A.Tremeau, « Numérisation et authentification d'œuvres d'art par vision numérique multicomposantes », journée thématique GDR Traitement Signal Image et Arts, CNAM Paris, 9 juin 2005.

[10] J.P.Chambard, S.Jaminion, «Evaluation de l'incertitude de mesure d'un capteur optique de numérisation 3D », 13e congrès international de métrologie, Juin 2007

[11] F. Larue, J.M. Dischler, "Efficient Registration and Calibration for Automatic Surface Light Field Acquisition", 7th VAST International Symposium on Virtual Reality, Archeology and Cultural Heritage, 2006

[12] M. Fournier, J.M. Dischler, D. Bechmann, « A new vector field distance transform and its application to mesh processing from 3D scanned data », to appear in The Visual Computer, 2007

[13] J.M. Dischler, F. Zara, « Real-time structured texture synthesis and editing using image-mesh analogies », The Visual Computer, Vol. 22(9), 2006

[14] O. Génevaux, F. Larue, J.M. Dischler, "Interactive Refraction on Complex Static Geometry using Spherical Harmonics", SI3D '06: Proceedings of the 2006 Symposium on Interactive 3D Graphics and Games, ACM Press, NY, USA - march 2006

[15] M. Fournier, J.M. Dischler, D. Bechmann, "3D distance transform adaptive filtering for smoothing and denoising triangle meshes", 4th International Conference on Computer Graphics and Interactive Techniques in Australasia and Southeast Asia (Graphite), 2006 